\title{
Electric-field modulation of the number of helical edge states in thin-film semiconductors
}

\author{
Zhan-Feng Jiang, Rui-Lin Chu, and Shun-Qing Shen* \\ Department of Physics, Center of Theoretical and Computational Physics, The University of Hong Kong, Pokfulam Road, Hong Kong
}

(Received 30 December 2009; revised manuscript received 15 February 2010; published 18 March 2010)

\begin{abstract}
We propose a method that can be used to modulate the topological orders or the number of helical edge states in ultrathin-film semiconductors without a magnetic field. By applying a staggered periodic potential, the system undergoes a transition from a topological trivial insulating state into a nontrivial one with helical edge states emerging in the band gap. Further study demonstrates that the number of helical edge states can be modulated by the amplitude and the geometry of the electric potential in a stepwise fashion, which is analogous to tuning the integer quantum Hall conductance by a magnetic field. We address the feasibility of experimental measurement of this topological transition.
\end{abstract}

DOI: 10.1103/PhysRevB.81.115322

PACS number(s): 73.43.-f, 73.50.-h, 85.75.-d

\section{INTRODUCTION}

Edge and surface physics arising from the topological insulators have been a major research focus in the condensedmatter physics recently. ${ }^{1-10}$ Usually spin-orbit coupling in these materials is so strong that the band gap between the conduction and valence bands can be inverted. In this case edge states or surface states emerge in the band gap, protected by time-reversal symmetry. The $Z_{2}$ invariant is established to govern the topological properties of both twodimensional (2D) and three-dimensional (3D) topological insulators. ${ }^{11,12}$ Searching for this topological state of quantum matter has been focused on specific materials such as $\mathrm{HgTe} / \mathrm{CdTe}$ quantum wells, ${ }^{4}$ bismuth alloys, ${ }^{7-10}$ and transition-metal oxide $\mathrm{Na}_{2} \mathrm{IrO}_{3} .{ }^{13}$ It was observed that there are five surface states in $\mathrm{Bi}_{1-x} \mathrm{Sb}_{x}$ (Ref. 7) and single Dirac cone in $\mathrm{Bi}_{2} \mathrm{Se}_{3}$ (Ref. 8) and $\mathrm{Bi}_{2} \mathrm{Te}_{3}$ (Ref. 10) while there is only a pair of helical edge states in $\mathrm{HgTe} / \mathrm{CdTe}$ quantum wells. ${ }^{4,14} \mathrm{Up}$ to now there is neither experimental report nor theoretical proposal to tune the topological number in these systems in a controllable way. It would be both theoretically and experimentally interesting if the topological number or the number of the helical edge states or surface states can be modulated like the quantum Hall conductance in a magnetic field.

In this paper, we propose a feasible approach to modulate consecutively the pair number of helical edge states by an electric means. To be specific, we consider a quasi-twodimensional semiconductor thin film or quantum well with strong spin-orbit coupling, which can be either topologically trivial or nontrivial. We apply a lateral surface superlattice (SSL) that creates a periodic potential on the film. It is found that the system changes from a trivial insulator into a quantum spin-Hall (QSH) insulator with helical edge states emerging at the sample edges. This phenomenon can be understood intuitively as the periodic potential splits the band structure into multiminibands, inverts the band gap and changes the $Z_{2}$ order of the bulk. However, unlike the QSH insulator, the band inversion story does not stop here. By further increasing the gate geometry or potential magnitude, the pair number of helical edge states goes up stepwise while the bulk band gap remains finite. In this manner we create a topological insulator whose edge states are tunable by purely electrical means.

\section{MODEL AND BAND FOLDING}

We start with an effective four-band Hamiltonian with the time-reversal invariance,

$$
H_{0}=\left(\begin{array}{cc}
h_{+}\left(-i \partial_{x},-i \partial_{y}\right) & 0 \\
0 & h_{-}\left(-i \partial_{x},-i \partial_{y}\right)
\end{array}\right),
$$

where $\quad h_{ \pm}=+D\left(\partial_{x}^{2}+\partial_{y}^{2}\right)+A\left(-i \partial_{x} \sigma_{y}+i \partial_{y} \sigma_{x}\right) \pm[\Delta / 2+B$ $\left.\left(\partial_{x}^{2}+\partial_{y}^{2}\right)\right] \sigma_{z}$ and $\sigma_{\alpha}$ are the Pauli matrices. The model was first introduced for the $\mathrm{HgTe} / \mathrm{CdTe}$ quantum well for $\mathrm{QSH}$ effect by Bernevig et al., ${ }^{3}$ and recently was derived for an ultrathin film of $3 \mathrm{D}$ topological insulator. ${ }^{15}$ The two cases have different basis although the forms are identical. $h_{+}$consists of the invariants in the irreducible representation $\bar{D}_{1 / 2}$ of SU(2) group. ${ }^{16}$ The whole model $H_{0}$ keeps the time-reversal invariance. $h_{+}$and $h_{-}$are the "time" reversal counterparts under the operation $\Theta=-i \sigma_{y} K$, where $K$ is the complex conjugate operator, $h_{+}=\Theta^{-1} h_{-} \Theta$. The block diagonalized form in $H_{0}$ allows us to study $h_{+}$and $h_{-}$separately and then put together to gain the physics for $H_{0}$. Additional terms such as those of bulk or structure inversion asymmetry can couple them together. Here we first assume that these effects are weak and negligible. All numerical calculations presented in Figs. 2-4 are for $h_{+}$. The results for $h_{-}$can be obtained by using time reversal operation.

The idea of a lateral SSL dates back to the 1970s. Various alternative techniques are known capable of creating SSLs. ${ }^{17}$ It is known that periodic potential created by SSLs induces Bloch minibands and minigaps on a two-dimensional electron gas (2DEG), which results in interesting transport behaviors. Consider this model on a SSL. To be specific and without loss of generality, we introduce a square-waveshaped periodic potential,

$$
V(y)=\left\{\begin{array}{cc}
V_{0} & 0 \leq y<d / 2 \\
-V_{0} & -d / 2 \leq y<0
\end{array}\right.
$$

and $V(y+d)=V(y)$. We write the Fourier series for $V(y)$ as $V(y)=\sum_{n} V_{n} e^{i n Q y}$ with the reciprocal vector $Q=2 \pi / d$ and the Fourier-transform component $V_{n}=2 i V_{0} / n \pi(n= \pm 1, \pm 3, \ldots)$. According to the Bloch theorem, ${ }^{18}$ the single-particle wave function of $h_{+}$for the band $\epsilon_{n, k}^{s}$ in this periodic potential $V(y)$ has the form $\Psi_{n}^{s}(x, y)=e^{i\left(k_{x} x+k_{y} y\right)} u_{n, k_{y}}^{s}(y)$ with $u_{n, k_{y}}^{s}(y)$ 


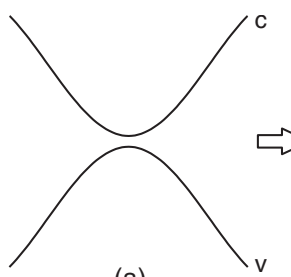

(a)

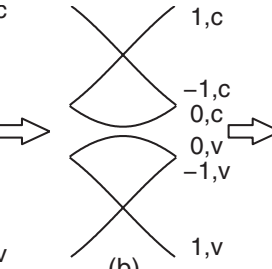

(b)

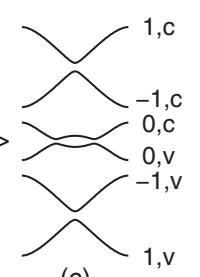

(c)
FIG. 1. Evolution of energy bands in a modulated periodic potential. (a) The band in the absence of periodic potential; (b) minibands formed in a periodic potential; (c) shifting of the minibands leads to the band inversion and band anticrossing.

$=u_{n, k_{y}}^{s}(y+d)$, where $k_{y}$ is confined in the first Brillouin zone (BZ) $k_{y} \in[-Q / 2,+Q / 2], n$ is an integer, and the superscript $s(=c, v)$ denotes the conduction $(c)$ and valence $(v)$ band. The wave function can be expressed as $u_{n, k_{y}}^{s}(y)$ $=\Sigma_{m} C_{n, k_{y}}^{s}(m) e^{i m Q y}$ with the coefficients $C_{n, k_{y}}$ determined by the central equation

$$
\left[h_{+}\left(k_{x}, m Q+k_{y}\right)-\epsilon_{n, k}^{s}\right] C_{n, k_{y}}^{s}(m)+\sum_{l} V_{l} C_{n, k_{y}}^{s}(m-l)=0
$$

with the condition $C_{n, k_{y}}^{s}(m)=\delta_{n, m} C_{n, k_{y}}^{s}(n)$ for $V=0$. In this way, the electron band is folded into many minibands confined in the first BZ as illustrated schematically in Figs. 1(a) and $1(\mathrm{~b})$, where the labels $(n, s)$ represent different minibands.

In the presence of $V$, the miniband structure can be obtained numerically by solving the central equation. It is found that when the potential $V$ increases the conduction minibands shift down and the valence minibands shift up, eventually the two bands cross and form a inverted band gap as shown in Fig. 1(c). Anticrossing between the two bands occurs, which is induced by the interaction term $A$. A simple perturbative viewpoint helps us to apprehend why the periodic potential $V$ tends to induce the band inversion. Take the band $(0, c)$ for our illustration. For a weak $V$, the calculation up to the second-order perturbation gives the energy correction for the band $(0, c)$,
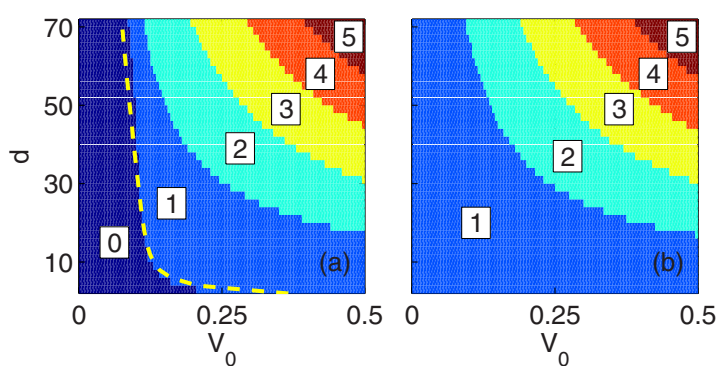

FIG. 2. (Color online) The phase diagram of Chern number in $\left(V_{0}, d\right)$ plane for the model of $h_{+}$with the Fermi energy $E_{f}=0$, (a) $\Delta / 2=0.01$ and (b) $\Delta / 2=-0.01$. The dashed line in (a) is obtained by solving Eq. (2) in the truncation approximation for $m=0$ and \pm 1 in $C_{m, k}^{s}$. Other parameters are $A=-B=1, D=0$, and the lattice space $a=1$. The Chern number will change a minus sign for the corresponding part of $h_{-}$.
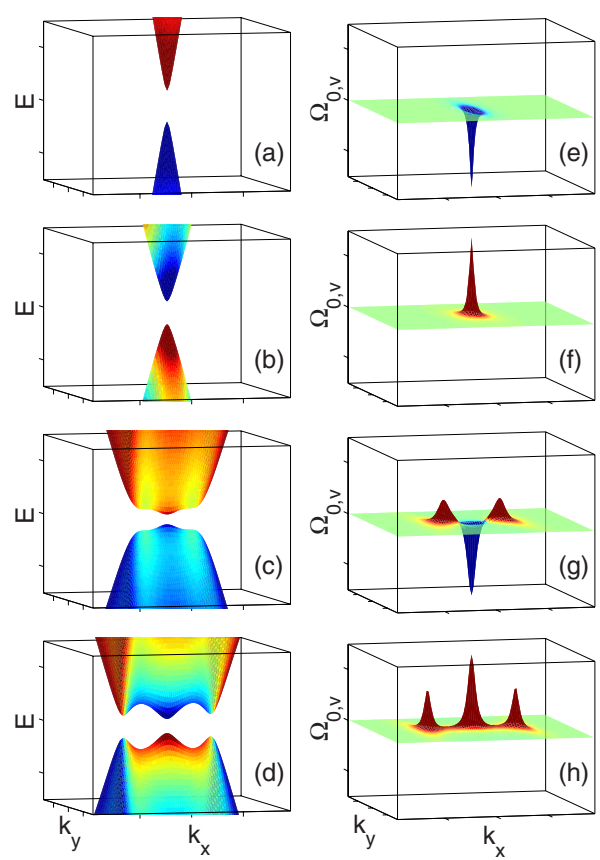

FIG. 3. (Color online) [(a)-(d)] Energy dispersion for the first valence and conduction band of $h_{+}$for various periodic potential strengths (a) $V_{0}=0.06$; (b) 0.14 ; (c) 0.23 ; and (d) 0.26 . Dark gray (red) indicates the dominant eigenstate is spin up, light gray (blue) indicates the dominant eigenstate is spin down. The other parameters are $\Delta / 2=0.01, d=30$. (a) and (b) corresponds to the transition of $C=0 \rightarrow 1$. (c) and (d) corresponds to the transition of $C=1$ $\rightarrow 2$. [(e)-(h)] Berry curvatures of the valence band around $\Gamma$ point corresponding to the band structures (a)-(d), respectively.

$$
\Delta \epsilon_{0, k}^{c}=\sum_{m, s, k_{y}^{\prime}} \frac{\mid\left\langle\left. u_{0, k_{y}}^{c}|V(\mathbf{r})| u_{m, k_{y}^{\prime}}^{s}\right|^{2}\right.}{\epsilon_{0, k}^{c}-\epsilon_{m, k_{y}^{\prime}}^{s}},
$$

here the summation excludes the band $(0, c)$ itself, $u_{m, k_{y}^{\prime}}^{s}$ and $\epsilon_{n, k}^{s}$ are the unperturbed eigenstate and eigenenergy of the miniband in the first BZ. Using the Fourier series of the potential, we have $\left\langle u_{0, k_{y}}^{c}|V(\mathbf{r})| u_{m, k_{y}^{\prime}}^{s}\right\rangle=V_{-m} \delta_{k_{y}, k_{y}}\left\langle u_{0, k_{y}}^{c} \mid u_{m, k_{y}}^{s}\right\rangle$. $V(\mathbf{r})$ couples the band $(0, c)$ and other conduction bands $(n, s=c)$ stronger than the valence bands since they come from same original bands and share the same spin indices. We have $\left|\left\langle u_{0, k_{y}}^{c} \mid u_{m, k_{y}}^{c}\right\rangle\right| \gg\left|\left\langle u_{0, k_{y}}^{c} \mid u_{m, k_{y}}^{v}\right\rangle\right|$. Thus we draw the conclusion that the perturbative correction is always negative, $\Delta \epsilon_{0, k}^{c}<0$, whose effect is shifting the band $(0, c)$ downward. Similarly, the valence band $(0, v)$ shifts upward in the field. At a certain value of $V$, the two bands cross and open a gap again, i.e., a negative one. Similar argument can be applied to other minibands. With increasing $V$, more and more minibands will cross near the zero-energy point. Thus the band inversion occurs consecutively because of the shifting of the minibands from the conduction and valence bands.

\section{MODULATION OF CHERN NUMBER}

The formation of the inverted band gap is of topological and experimental interests. To gain a quantitative insight of 

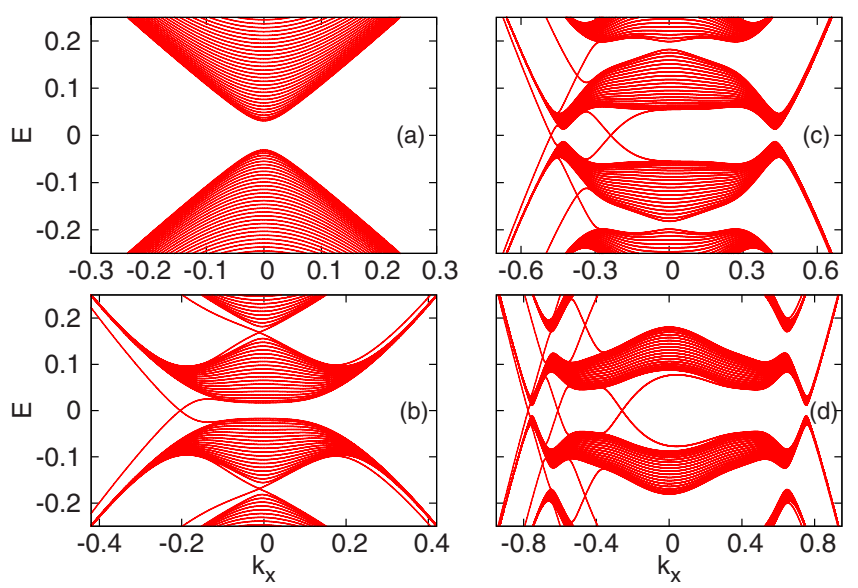

FIG. 4. (Color online) Band structure evolution of a strip geometry in a periodic potential for $h_{+}$. Strip width 400, $d=20$, and $\Delta / 2=0.03$. (a) $V=0, C=0$; (b) $V_{0}=0.3, C=1$; (c) $V_{0}=0.6, C=2$; and (d) $V_{0}=1.0, C=3$. Asymmetric dispersion for edge state originates from the symmetric breaking of the potential on the strip with open boundary condition.

this physical picture, we discretize the Hamiltonian $h_{+}(\boldsymbol{k})$ in Eq. (2) on a square lattice. To have a band insulator in $h_{+}(\boldsymbol{k})$, it is required that $|B|>|D|$. For the purpose of numerical calculation, we make the parameters material independent by setting $A=-B=1, D=0$, and the lattice space $a=1$. Other choices such as nonzero $D$ or $A \neq-B$ will not change the conclusion in the present work qualitatively. Eigenenergies and eigenstates are solved numerically for the periodic system. Following Thouless et al. ${ }^{19}$ we come to calculate the Hall conductance of $h_{+}$in the band gap, which is equivalent to the Chern number of the filled valence bands. The Berry curvature for each band is defined as ${ }^{20}$

$$
\Omega_{n, s}(k)=i\left(\left\langle\frac{\partial u_{n, k}^{s}}{\partial k_{x}} \mid \frac{\partial u_{n, k}^{s}}{\partial k_{y}}\right\rangle-\left\langle\frac{\partial u_{n, k}^{s}}{\partial k_{y}} \mid \frac{\partial u_{n, k}^{s}}{\partial k_{x}}\right\rangle\right) .
$$

The first Chern number in the band gap is then computed by summing up Berry curvatures of the occupied bands and integrate over the first BZ $C=\frac{1}{2 \pi} \Sigma_{n} \iint d^{2} k \Omega_{n, v}(k)$. The Hall conductance for $h_{+}$is related to the first Chern number by $\sigma_{x y}=\frac{e^{2}}{h} C{ }^{19,21}$ In the absence of the potential, the Chern number was found to be $C=-[\operatorname{sgn}(\Delta)+\operatorname{sgn}(B)] / 2,{ }^{15}$ which is in agreement with the existence condition of solution of edge states in the Schrödinger equation in Eq. (1). ${ }^{22}$ The signs of the two model parameters in the term $\left(\Delta / 2-B k^{2}\right) \sigma_{z}$ of $h_{+}$ determine the value of $C, 0$, or \pm 1 , and further whether the system is topologically trivial or not. In the presence of $V$, it is found that the Chern number changes by 1 when the valence and conduction bands cross. By continually changing the potential period $d$ and potential magnitude $V_{0}$, we obtain a phase diagram for the Chern number in Fig. 2 with the numbers in the boxes indicating the values. We see that in this system the Chern number is a function of the potential period $d$ and amplitude $V_{0}$.

Examination of the evolution of the band structure and Berry curvature at the transition courses further confirms our claim. In Fig. 3 we show the first two transitions from $C$
$=0$ to 1 , and from $C=1$ to 2 . By increasing $V_{0}$, the band gap closes and reopens in Figs. 3(a) and 3(b). Correspondingly, the Berry curvature peak become sharp, and reverses its value dramatically, which accounts for the change in the Chern number. At the corresponding $\boldsymbol{k}$ points, the eigenwave functions also switch their band indexes quickly. By further increasing $V_{0}$, the peak of the Berry curvature splits into two subpeaks, and the third peak with an opposite sign grows up while the Chern number remain to be $C=1$. In the second transition, the third peaks reverses again just like the first transition, and the Chern number changes from $C=1$ to 2 . Therefore accompanied by every "closing and reopening" of the band gap the Chern number changes by 1 .

\section{EDGE STATES AND SPECTRUM}

According to the edge-bulk correspondence, ${ }^{23,24}$ the first Chern number determines number of the edge states when the system has an open boundary. We take a strip geometry that is parallel to the $x$ direction with open boundary condition along the $y$ direction. We start from a topological trivial case of $\Delta>0$ and $B<0$, and scrutinize its changing in the band structure when varying the periodic potential $V_{0}$. In the absence of the potential $V$ the system is insulating and topologically trivial indicated by a positive band gap. As $V_{0}$ increases, the conduction bands shift down and valence bands shift up. Eventually the two bands cross and a band gap reopens. A pair of edge states appears connecting the valence and conduction bands as shown in Fig. 4(b). Detailed analysis shows that the wave functions of these states indeed reside on the sample edges only. As $V_{0}$ goes further up, more bands cross and more pairs of edge states are formed at distinct $\boldsymbol{k}$ points as shown in Figs. 4(c) and 4(d). It is interesting to observe that the band gap at $E=0$ always retains a finite value at certain stages throughout the evolution. We can see the appearance of the edge states corresponds to the change in the Chern number from $C=0$ to $C=3$ by comparing Figs. 3 and 4 . Thus modulation of edge-state numbers is clearly reflected in the step wise changing of the Chern numbers.

$h_{+}$and $h_{-}$are the time-reversal counterparts of each other. Each edge state $\left|\psi_{+}\right\rangle$in $h_{+}$has a counterpart $\left|\psi_{-}\right\rangle=\Theta\left|\psi_{+}\right\rangle$in $h_{-}$and they form a pair of helical edge states. As a result, the large Chern number $(C>1)$ indicates multiple pairs of helical edge states in the system $H_{0}$ in this periodic potential $V$. For a thin film fabricated on the substrate, the top-bottom symmetry will be broken due to the interface of the thin film and the substrate. This fact will remove the degeneracy of the spectra from $h_{+}$and $h_{-}$. An off-diagonal term of structural inversion asymmetry is added to the model $H_{0}$ in Eq. (1), ${ }^{25}$ $\Delta V=V_{S I A}\left(\begin{array}{cc}0 & \sigma_{0} \\ \sigma_{0} & 0\end{array}\right)$ which connects the up and down blocks ( $\sigma_{0}$ is the $2 \times 2$ identity matrix). Similar physics happens if the sample breaks the bulk inversion symmetry in quantum wells. ${ }^{26}$ In the presence of $V_{\text {SIA }}$, numerical calculation still demonstrates existence of the edge states in the four-band model, which is characteristic of QSH phase. Thus this term does not destroy the QSH phase explicitly.

\section{DISCUSSION AND CONCLUSION}

Feasibility of experimental realization of this phenomenon depends on spin decoherence length in the sample and 
the fabrication of the periodic potential. The spin decoherence length was estimated to be $1-2 \mu \mathrm{m}$ in the $\mathrm{HgTe} / \mathrm{CdTe}$ quantum well ${ }^{4,14}$ and expected to be longer in a thin film of $\mathrm{Bi}_{2} \mathrm{Se}_{3} \cdot{ }^{25}$ On the periodicity of the modulated electric field, the sub-100-nm period of SSL fabrications was reported a decade ago, ${ }^{27}$ and the $3 \mathrm{~nm}$ periodicity for a graphene on $\mathrm{Ru}(111)$ recently. ${ }^{28}$ For a set of realistic parameters for $\mathrm{HgTe} /$ CdTe quantum well with a normal band gap $\Delta=4 \mathrm{meV},{ }^{3}$ the magnitude of the potential with periodicity $d=50 \mathrm{~nm}$ is calculated to $V_{0}=40 \mathrm{meV}$ for the transition of $C=0-1$ and $V_{0}$ $=110 \mathrm{meV}$ for the transition of $C=1-2$. We speculate that the modern techniques of superlattice make this electric-field modulation possible.

In summary, the band structure of a thin film or quantum well are folded into the minibands in the reduced BZ by a periodic potential and can be modulated such that the conduction bands shift downward and the valence bands shift upward. Each process of the band gap closing and reopening will change the Chern number by 1 . As a result the number of the helical edge states will increase or decrease by 1 . This demonstrates the possibility of the electric-field modulation of topological orders in the thin-film semiconductors, which is analogous to the integer quantum Hall effect in a strong magnetic field. Direction of electrons of the helical edge states is of interests in quantum information and quantum processing. Controllable number of the helical edge states will pave an alternative route for application of edge-state physics in the future.

\section{ACKNOWLEDGMENT}

This work was supported by the Research Grant Council of Hong Kong under Grants No. HKU 7041/07P and No. HKU 10/CRF/08. *sshen@hkucc.hku.hk

${ }^{1}$ For introductions to topological insulators, see S. C. Zhang, Phys. 1, 6 (2008); M. Buttiker, Science 325, 278 (2009); J. Moore, Nat. Phys. 5, 378 (2009).

${ }^{2}$ C. L. Kane and E. J. Mele, Phys. Rev. Lett. 95, 226801 (2005).

${ }^{3}$ B. A. Bernevig, T. L. Hughes, and S. C. Zhang, Science 314, 1757 (2006).

${ }^{4}$ M. König, S. Wiedmann, C. Brüne, A. Roth, H. Buhmann, L. W. Molenkamp, X. L. Qi, and S. C. Zhang, Science 318, 766 (2007).

${ }^{5}$ J. Li, R. L. Chu, J. K. Jain, and S. Q. Shen, Phys. Rev. Lett. 102, 136806 (2009).

${ }^{6}$ L. Fu, C. L. Kane, and E. J. Mele, Phys. Rev. Lett. 98, 106803 (2007).

${ }^{7}$ D. Hsieh, D. Qian, L. Wray, Y. Xia, Y. S. Hor, R. J. Cava, and M. Z. Hasan, Nature (London) 452, 970 (2008).

${ }^{8}$ Y. Xia, D. Qian, D. Hsieh, L. Wray, A. Pal, H. Lin, A. Bansil, D. Grauer, Y. S. Hor, R. J. Cava, and M. Z. Hasan, Nat. Phys. 5, 398 (2009).

${ }^{9}$ H. Zhang, C. X. Liu, X. L. Qi, X. Dai, Z. Fang, and S. C. Zhang, Nat. Phys. 5, 438 (2009).

${ }^{10}$ Y. L. Chen, J. G. Analytis, J.-H. Chu, Z. K. Liu, S.-K. Mo, X. L. Qi, H. J. Zhang, D. H. Lu, X. Dai, Z. Fang, S. C. Zhang, I. R. Fisher, Z. Hussain, and Z.-X. Shen, Science 325, 178 (2009).

${ }^{11}$ C. L. Kane and E. J. Mele, Phys. Rev. Lett. 95, 146802 (2005).

${ }^{12}$ J. E. Moore and L. Balents, Phys. Rev. B 75, 121306(R) (2007).

${ }^{13}$ A. Shitade, H. Katsura, J. Kuneš, X. L. Qi, S. C. Zhang, and N. Nagaosa, Phys. Rev. Lett. 102, 256403 (2009).

${ }^{14}$ A. Roth, C. Brune, H. Buhmann, L. W. Molenkamp, J. Maciejko, X. L. Qi, and S. C. Zhang, Science 325, 294 (2009).
${ }^{15}$ H. Z. Lu, W. Y. Shan, W. Yao, Q. Niu, and S. Q. Shen, Phys. Rev. B 81, 115407 (2010).

${ }^{16} \mathrm{R}$. Winkler, Spin-Orbit Coupling Effects in Two-Dimensional Electron and Hole Systems (Springer, Berlin, 2003), p. 64.

${ }^{17}$ R. Tsu, Superlattice to Nanoelectronics (Elsevier, Oxford, 2005).

${ }^{18}$ C. Kittel, Introduction to Solid State Physics, 7th ed. (Wiley, New York, 1996), Chap. 7, p. 183.

${ }^{19}$ D. J. Thouless, M. Kohmoto, M. P. Nightingale, and M. den Nijs, Phys. Rev. Lett. 49, 405 (1982).

${ }^{20}$ M. C. Chang and Q. Niu, J. Phys.: Condens. Matter 20, 193202 (2008); D. Xiao, M. C. Chang, and Q. Niu, arXiv:0907.2021 (unpublished).

${ }^{21}$ M. Kohmoto, Ann. Phys. (N.Y.) 160, 343 (1985).

${ }^{22}$ B. Zhou, H. Z. Lu, R. L. Chu, S. Q. Shen, and Q. Niu, Phys. Rev. Lett. 101, 246807 (2008).

${ }^{23}$ Y. Hatsugai, Phys. Rev. Lett. 71, 3697 (1993).

${ }^{24}$ X. L. Qi, Y. S. Wu, and S. C. Zhang, Phys. Rev. B 74, 045125 (2006).

${ }^{25}$ Y. Zhang, K. He, C. Z. Chang, C. L. Song, L. L. Wang, X. Chen, J. F. Jia, Z. Fang, X. Dai, W. Y. Shan, S. Q. Shen, Q. Niu, X. L. Qi, S. C. Zhang, X. C. Ma, and Q. K. Xue, arXiv:0911.3706, Nat. Phys. (to be published); W. Y. Shan, H. Z. Lu, and S. Q. Shen, arXiv:1001.0526 (unpublished).

${ }^{26}$ M. König, H. Buhmann, L. W. Molenkamp, T. Hughes, C. X. Liu, X. L. Qi, and S. C. Zhang, J. Phys. Soc. Jpn. 77, 031007 (2008).

${ }^{27}$ A. Messica, A. Soibel, U. Meirav, A. Stern, H. Shtrikman, V. Umansky, and D. Mahalu, Phys. Rev. Lett. 78, 705 (1997).

${ }^{28}$ S. Marchini, S. Gunther, and J. Wintterlin, Phys. Rev. B 76, 075429 (2007). 